\title{
CHARACTERIZATIONS FOR THE GENERALIZED FRACTIONAL INTEGRAL OPERATORS ON MORREY SPACES
}

\author{
ERIDANi, HENDra GunAWAn, EIICHI NAKAi AND YoshiHIRO SAWANO
}

Abstract. We present some characterizations for the boundedness of the generalized fractional integral operators on Morrey spaces. The characterizations follow from two key estimates, one for the norm of some functions in Morrey spaces, and another for the values of the corresponding fractional integrals. We prove three theorems about necessary and sufficient conditions. We show that these theorems are independent by giving some examples. We also obtain counterparts for the weak generalized Morrey spaces.

Mathematics subject classification (2010): Primary 42B35; Secondary 26A33, 46E30, 42B20, 43A15. Keywords and phrases: Fractional integrals, bounded operators, Morrey spaces.

\section{REFERENCES}

[1] D. R. AdAms, A note on Riesz potentials, Duke Math. J. 42 (1975), 765-778.

[2] W. S. BUDHI AND J. LINDIARTI, Boundedness of multilinear generalized fractional integral operators in generalized Morrey space, Far East J. Math. Sci. 57, 1 (2011), 91-104.

[3] V. Burenkov And V. Guliyev, Necessary and sufficient conditions for the boundedness of the Riesz potential in local Morrey-type spaces, Potential Anal. 30, 3 (2009), 211-249.

[4] V. I. Burenkov, A. Gogatishvili, V. S. Guliyev and R. Ch. Mustafayev, Boundedness of the Riesz potential in local Morrey-type spaces, Potential Analysis 35, 1 (2011), 67-87.

[5] F. Chiarenza And M. Frasca, Morrey spaces and Hardy-Littlewood maximal function, Rend. Mat. 7 (1987), 273-279.

[6] ERIDAni, H. Gunawan, AND E. NAKai, On generalized fractional integral operators, Sci. Math. Jpn. 60 (2004), 539-550.

[7] ERIDANi, M. I. UtOYo, AND H. GUNAWAN, A characterization for fractional integral operators on generalized Morrey spaces, Anal. Theory Appl. 28, 3 (2012), 263-267.

[8] ERIDANi AND Y. Sawano, Fractional Integral Operators in Generalized Morrey Spaces Defined on Metric Measure Spaces, Proc. A. Razmadze Math. Inst. 158 (2012), 13-24.

[9] ERIdani, AND M. I. Utoyo, A note on Nakai's results, Uzbek. Mat. Zh. 4 (2009), 172-179.

[10] A. D. GADJIEV, On generalized potential-type integral operators, Dedicated to Roman Taberski on the occasion of his 70th birthday, Funct. Approx. Comment. Math. 25 (1997), 37-44.

[11] V. S. GuliYeV, Boundedness of the maximal, potential and singular operators in the generalized Morrey spaces, Journal of inequalities and applications, Volume 2009, Article ID 503948, 20 pages.

[12] V. S. Guliyev, S. S. Aliyev, T. Karaman, And P. S. Shukurov, Boundedness of sublinear operators and commutators on generalized Morrey spaces, Integral Equations and Operator Theory 71, Issue 3 (2011), 327-355.

[13] V. S. GuliYev, J. HaS AnOv, AND Y. ZEREn, Necessary and sufficient conditions for the boundedness of the Riesz potential in modified Morrey spaces, J. Math. Inequal. 5, 4 (2011), 491-506.

[14] V. S. Guliyev, S. S. Aliyev, and R. Ch. Mustafayev, On generalized fractional integrals, Trans. Acad. Sci. Azerb. Ser. Phys.-Tech. Math. Sci. 21, 4 (2001), Math. Mech., 63-71, 237.

[15] V. S. Guliyev, AND R. CH. Mustafayev, On generalized fractional integrals on spaces of homogeneous type, Khazar Journal of Mathematics 2, 2 (2006), 49-60. 
[16] V. S. Guliyev, And R. Mustafayev, Boundedness of the anisotropic maximal and anisotropic singular integral operators in generalized Morrey spaces, Acta Math. Sin. (Engl. Ser.) 27, 12 (2011), 2361-2370.

[17] V. S. GULIYEV, AND Y. SAWANo, Linear operators on Generalized Morrey spaces with non-doubling measures, to appear in Publ. Math. Debrecen.

[18] H. Gunawan, A note on the generalized fractional integral operators, J. Indones. Math. Soc. 9 (2003), 39-43.

[19] H. GUNAWAN, AND ERIDANI, Fractional integrals and generalized Olsen inequalities, Kyungpook Math. J. 49 (2009), 31-39.

[20] K. KuRATA, AND S. SugAno, A remark on estimates for uniformly elliptic operators on weighted $L^{p}$ spaces and Morrey spaces, Math. Nachr. 209 (2000), 137-150.

[21] L. Liguang, Y. Sawano, And D. YAng, Morrey-type Spaces on Gauss Measure Spaces and Boundedness of Singular Integrals, J. Geometrical Analysis, online.

[22] Y. Mizuta, E. NAKAI, T. OHNO, AND T. Shimomura, Boundedness of fractional integral operators on Morrey spaces and Sobolev embeddings for generalized Riesz potentials, J. Math. Soc. Japan 62, 3 (2010), 707-744.

[23] Y. Mizuta, E. NaKai, T. Ohno, And T. Shimomura, An elementary proof of Sobolev embeddings for Riesz potentials of functions in Morrey spaces $L^{1, v, \beta}(G)$, Hiroshima Math. J. 38, 3 (2008), 425436.

[24] Y. Mizuta, E. NAKai, Y. Sawano, AND T. Shimomura, Gagliardo-Nirenberg inequality for generalized Riesz potentials of functions in Musielak-Orlicz spaces, Arch. Math. 98, 3 (2012), 253263.

[25] E. NAKAI, Hardy-Littlewood maximal operator, singular integral operators and the Riesz potentials on generalized Morrey spaces, Math. Nachr. 166 (1994), 95-103.

[26] E. NAKAI, A characterization of pointwise multipliers on the Morrey spaces, Sci. Math. 3 (2000), 445-454.

[27] E. NAKAI, On generalized fractional integrals, Taiwanese J. Math. 5 (2001), 587-602.

[28] E. NAKAI, On generalized fractional integrals on the weak Orlicz spaces, $\mathrm{BMO}_{\varphi}$, the Morrey spaces and the Campanato spaces, Function spaces, interpolation theory and related topics (Lund, 2000), 389-401, de Gruyter, Berlin, 2002.

[29] E. NAKAI, Orlicz-Morrey spaces and the Hardy-Littlewood maximal function, Studia Math. 188, 3 (2008), 193-221.

[30] E. NAKAI, A generalization of Hardy spaces $H^{p}$ by using atoms, Acta Math. Sin. (Engl. Ser.) 24, 8 (2008), 1243-1268.

[31] E. Nakai And G. Sadasue, Martingale Morrey-Campanato spaces and fractional integrals, J. Funct. Spaces Appl. 2012, Art. ID 673929, 29 pp.

[32] J. PeEtre, On convolution operators leaving $L^{p, \lambda}$ spaces invariant, Ann. Mat. Pura Appl. 72 (1966), 295-304.

[33] J. Peetre, On the theory of $\mathscr{L}_{p, \lambda}$ spaces, J. Funct. Anal. 4 (1969), 71-87.

[34] Y. SAWANO, $l^{q}$-valued extension of the fractional maximal operators for non-doubling measures via potential operators, International Journal of Pure and Applied Mathematics 26, 4 (2006), 505-523.

[35] Y. SAWANo, Generalized Morrey Spaces for non-doubling measures, Nonlinear differ. equ. appl. 15 (2008), 413-425.

[36] Y. Sawano And T. Shimomura, Sobolev embeddings for Riesz potentials of functions in nondoubling Morrey spaces of variable exponents, Collectanea Mathematica, in press.

[37] Y. SAWANo AND S. SHIRAI, Compact commutators on Morrey spaces with non-doubling measures, Georgian Math. J. 15, 2 (2008), 353-376.

[38] Y. SAWANO, S. SUgAnO, AND H. TANAKA, A note on generalized fractional integral operators on generalized Morrey spaces, Bound. Value Probl. 2009, Art. ID 835865, 18 pp.

[39] Y. SAWANO, S. SUGANO, AND H. TANAKA, Generalized fractional integral operators and fractional maximal operators in the framework of Morrey spaces, Trans. Amer. Math. Soc. 363, 12 (2011), 64816503.

[40] Y. Sawano, S. Sugano And H. TAnaKa, Orlicz-Morrey spaces and fractional operators, Potential Anal. 36, 4 (2012), 517-556.

[41] Y. SAWANO AND H. WADADE, On the Gagliardo-Nirenberg type inequality in the critical SobolevMorrey space, J. Fourier Anal. Appl., online. 
[42] I. SihwANingRum, S. MARYANI, AND H. GunAwan, Weak type inequalities for fractional integral operators on generalized non-homogeneous Morrey spaces, Anal. Theory and Appl. 28, 1 (2012), $65-72$.

[43] E. M. SteIn, Singular Integrals and Differentiability Properties of Functions, Princeton University Press, Princeton, New Jersey, 1970.

[44] E. M. STEIn, Harmonic Analysis: Real Variable Methods, Orthogonality, and Oscillatory Integrals, Princeton University Press, Princeton, New Jersey, 1993.

[45] S. Sugano, Some inequalities for generalized fractional integral operators on generalized Morrey spaces, Math. Inequal. Appl. 14, 4 (2011), 849-865.

[46] S. Sugano And H. TANAKA, Boundedness of fractional integral operators on generalized Morrey spaces, Sci. Math. Jpn. 58, 3 (2003), 531-540 (Sci. Math. Jpn. Online 8 (2003), 233-242). 